\title{
BMC Sports science, medicine, and rehabilitation reviewer acknowledgement 2014
}

\author{
Clare Partridge
}

\section{Contributing reviewers}

The editors of Sports Science, Medicine and Rehabilitation would like to thank all our reviewers who have contributed to the journal in Volume 6 (2014).

\section{Simon Angus}

Australia

Francisco Areces Corcuera

Spain

Julien Steven Baker

UK

Arnhild Bakken

Qatar

Tiago Barbosa

Singapore

Alan Barker

UK

Alan Batterham

UK

Tom Bauer

USA

Britton Brewer

USA

Aurelien Bringard

Switzerland

Steven Broglio

USA

Martin Burtscher

Austria

Michael Carmont

UK

\author{
Spain \\ Karly Oi Wan Chan \\ China \\ Chi Ching Gary Chow \\ Hong Kong \\ James Clark \\ USA \\ Jamie Cooper \\ USA \\ Jennifer Copeland \\ Canada \\ Mario Costa \\ Portugal \\ Carlos Crestani \\ Brazil
}

Adelaida María Castro-Sánchez

Antonio Crisafulli

Italy

Aaron Daluiski

USA

Fred Dimenna

USA

Catherine Disselhorst-Klug

Germany

Lars Donath

Switzerland
Jacques Duysens

Netherlands

Pascal Edouard

France

Mikel Egana

Ireland

Brandon Erickson

USA

Jonathan Esteve-Lanao

Spain

Gertjan Ettema

Norway

Hani Fadel

Saudi Arabia

Abdulaziz Farooq

Qatar

Oliver Faude

Switzerland

Catherine Fieseler

USA

Richard Foster

UK

Reginaldo Fukuchi

Brazil

Alejandro Galan-Mercant

Spain

Correspondence: clare.partridge@biomedcentral.com

BioMed Central, Floor 6, 236 Gray's Inn Road, London WC1X 8HB, UK 


\author{
Laura Goldberg \\ USA
}

Eric Goulet

Canada

\section{Mathieu Gourlan}

France

Freedom Nkhululeko Gumedze

South Africa

Maria Hagstromer

Sweden

Brian Hanley

UK

Marquis Hawkins

USA

Stewart Head

Australia

Jose M Heredia Jimenez

Spain

Angela Hibbs

UK

John Hill

USA

Jonathan Hoffman

Israel

\section{Kuno Hottenrott}

Germany

Wen-Hao Hsu

USA

John Timothy Inglis

Canada

Athanasios Jamurtas

Greece

John Kalbfleisch

USA

\section{Alon Kalron \\ Israel}

Michelle Keightley

Canada

Michael Kingsley

Australia

Beat Knechtle

Switzerland

Kevin Kwong

Hong Kong
Joyce Cy Leung

Hong Kong

Carl Lombard

South Africa

Wings Tjing Yung Loo

Hong Kong

Reidar Lystad

Australia

Jelena Macan

Croatia

Duncan Macfarlane

Hong Kong

Fabiana Machado

Brazil

Zuzana Machotka

Australia

Nicola Maffulli

UK

Daniel Marinho

Portugal

Amy Mark

Canada

Fulvio Marzatico

Italy

Kei Masani

Canada

Michele Mauro

Italy

Alan McCall
France
Teri McCambridge
USA
Suzanne McDonough
UK
Chris McLellan
Australia
Jose Antonio Merchán-Baeza
Spain
Neil Messenger
UK
Guillaume Millet
Canada
Tony Monnet
France

Kevin Netto

Australia

Georg Neumann

Germany

Zhen Ni

Canada

Pantelis Nikolaidis

Greece

David Oxborough

UK

Adriana Perez

USA

Maria Francesca Piacentini

Italy

Pierre Portero

France

Geoff Power

Canada

Craig Ranson

UK

Christoph Raschka

Germany

Charles Rice

Canada

Gordon Robertson

Canada

Jose M Saavedra

Spain

Daniel Sánchez-Zuriaga

Spain

Jonathon Senefeld

USA

Mariano Serrao

Italy

Sameer Shah

USA

Jasper Shealy

USA

Tzyy-Yuang Shiang

Taiwan

Eric Stöhr

UK

Antoni Sureda

Spain 
Wolfgang Taube

Switzerland

Pedro Tauler

Spain

Danny Taylor

UK

Daniel Theisen

Luxembourg

Panos Thomas

UK

Mike Tipton

UK
Takuro Tobina

Japan

Tamara Valovich Mcleod

USA

Vincent Van Hees

UK

Cahouet Violaine

France

Feng Wei

USA

Jeffrey Willardson

USA
Jonathan Williams

UK

Junjie Xiao

China

Ewa Ziemann

Poland

Hassane Zouhal

France

Received: 8 January 2015 Accepted: 8 January 2015 Published: 18 February 2015

doi:10.1186/2052-1847-7-6

Cite this article as: Partridge: BMC Sports

science, medicine, and rehabilitation

reviewer acknowledgement 2014. BMC Sports

Science, Medicine, and Rehabilitation 2015 7:6. 